\title{
Postural responses to stationary and moving scenes as a function of distance to the scene ${ }^{*}$
}

\author{
T.M.H. Dijkstra, C.C.A.M. Gielen and B.J.M. Melis \\ Unilersity of Nijmegen, Nijmegen, The Netherlands
}

\begin{abstract}
Dijkstra, T.M.H., C.C.A.M. Gielen and B.J.M. Melis, 1992. Postural responses to stationary and moving scenes as a function of distance to the scene. Human Movement Science 11, 195-203.

Amplitude of postural sway in fore-after direction was investigated as a function of suggested distance between the observer and a stationary or moving scene. These experiments were done with a set-up in which movements of the observer were fed back real-time to a graphics workstation to modify the video image projected on the screen. The results show that sway amplitude for a stationary scene increases with increasing distance. Sway amplitude in response to a scene moving along the line of sight with constant amplitude did not depend on distance. Both of these results can be explained by assuming a threshold for visual motion perception and by assuming that observers move so as to minimize retinal slip.
\end{abstract}

\section{Introduction}

Vision provides an important source of information for the control of posture (see e.g. Dichgans and Brandt 1978). This can easily be understood since changes in position of the head yield a deformation of the projection of the environment on the retina. If the environment has enough structure, this deformation of the visual scene contains all information necessary to detect direction and amplitude of the movement relative to the environment. This presumably explains why

\footnotetext{
* This research was supported by the European Community in the ESPRIT 2, BASIC RESEARCH Program MUCOM, project number 3149. The authors gratefully acknowledge the support from the Dutch Foundation for Biophysics.

Requests for reprints should be sent to T.M.H. Dijkstra, Dept. of Medical Physics and Biophysics, University of Nijmegen, Geert Grootplein Noord 21, 6525 EZ Nijmegen, The Netherlands.
} 
postural sway in the dark is at least twice as large as in an illuminated environment (Paulus et al. 1989; Van Asten et al. 1988a,b).

It has been reported that the distance of an observer relative to a particular scene is an important parameter. When postural sway of a standing subject is measured as a function of the distance to a stationary scene, sway amplitude increases with increasing distance (Brandt et al. 1985; Paulus et al. 1989). The explanation of this phenomenon provided by virtually all theories on optic flow is that the retinal displacement of the stationary scene due to a movement of the observer decreases with increasing distance to the scene. As a consequence, visual locking to the environment is easier when the scene is close to the subject.

Since the retinal displacement induced by a displacement of a textural element decreases with distance to the observer, it might be expected that when an observer is confronted with a scene moving with a constant amplitude at various distances of the observer, the postural response decreases with distance to the scene. This presupposes that the amplitude of the moving scene is the same for all distances. This would imply that responses to displacement of a scene are not invariant with respect to distance of the scene. Such relationships might then be related to the earlier finding (Gielen and Van Asten 1990) that postural sway in response to simulated motion of a 3-dimensional tunnel was not invariant with respect to the direction of gaze.

However, it has been shown (Brandt et al. 1975) that when an observer is confronted with two vertical scenes rotating around the line of sight at different distances, the far scene dominates postural responses over the near scene. This result suggests that distance towards the scene is somehow incorporated in the evaluation of optic flow. Thus, it may be that postural responses to simulated motion of a scene with constant amplitude are invariant for the distance to the scene. The aim of this paper is to investigate how postural sway depends on the distance to a moving scene.

\section{Methods}

Subjects

Three subjects with normal or corrected-to-normal vision were tested in the experiment with the stationary scene and five in the 
experiment with the moving scene. All subjects were familiar with the purpose of the experiment. Subjects were instructed to look at the centre of the stimulus and to assume a relaxed posture. Subjects stood on firm stable support in a normal Romberg posture.

\section{Experimental set-up}

Visual stimuli with a spatial resolution of $1152 \times 900$ pixels were generated with a SUN4/260 CXP workstation. The stimuli were projected on a large translucent screen (dimensions $2.5 \times 2$ meter) with a BARCO video projection system (GRAPHICS 400 ). The frame rate was $66 \mathrm{~Hz}$. The translucent screen was homogeneously white without any visible texture.

The subject wore a pair of goggles which limited the angle of the viewing area to about 110 deg rotationally symmetric for the left eye. The right eye was completely covered. Due to the restriction of the viewing angle, the edge of the screen was not visible to the subject. In this way all visible cues that could provide a reference to the stable environment were eliminated.

The subject stood in front of the screen wearing a helmet on which 5 infra-red light emitting diodes (IRED's) were mounted. The positions of these IRED's were measured with a WATSMART system at a sampling rate of $100 \mathrm{~Hz}$. The 2-D coordinates from the two camera's of the WATSMART system were converted in real-time in 3-D coordinates. The 3-D coordinates were sent to the SUN4 under DMA control. The SUN4 was programmed to generate a video image of a moving scene, viewed from a particular position. With this set-up each new frame (every $15 \mathrm{~ms}$ ) was a new image of the simulated moving 3-D scene from the subjects's point of view. These points of view were calculated using the average translation of the IRED's and were saved for later analysis. The total delay in the feed-back loop between movement of the observer and update of the video image for that movement was close to $30 \mathrm{~ms}$. No detailed experiments are known as to which delay is acceptable. Some indication can be gained from the study by Brandt et al. (1985) who saw no significant effect on posture for stroboscopic illumination at frequencies higher than $16 \mathrm{~Hz}$. This indicates that a delay of $30 \mathrm{~ms}$ is small enough for the purpose of this study. 
In these experiments the visual stimuli simulated a vertical dark wall covered with 300 green dots each with a dimension of $0.2 \times 0.2$ degrees. The dots had a uniform density per solid angle as seen from the average viewing position and lay between 10 degrees and 40 degrees eccentricity. Thus the dot density was $0.26 \%$ and was constant for all conditions. This is important since recent experiments (Howard and Heckmann 1989) have shown that the conclusion that a distant scene has more influence over vection than a near scene, cannot be made unequivocally without considering the relative sizes of the displays and the motion contrast between the near and far display. The stimulus was centred around the line of sight. In the centre of the stimulus there were no dots shown because of aliasing effects. The subjects did not have the impression that the scene moved independently of them, i.e. all visual motion was perceived as ego-motion.

The screen was always viewed from a distance of $50 \mathrm{~cm}$, thereby eliminating all distance cues except for accommodation and the relation between visual motion and head motion (an action-perception cue). Accommodation has been shown to be a very weak distance cue (Fisher and Ciufredda 1988) which leaves only the action-perception cue.

In the first experiment the scene was stationary. The dots on the screen only moved as a result of the feedback. Postural response was measured for the suggested distances of $10 \mathrm{~cm}, 20 \mathrm{~cm}, 40 \mathrm{~cm}, 80 \mathrm{~cm}$ and $110 \mathrm{~cm}$. In the second experiment the scene was sinusoidally driven with an amplitude of $2 \mathrm{~cm}$ and a frequency of $0.2 \mathrm{~Hz}$. Postural response was measured for the suggested distances of $25 \mathrm{~cm}, 50 \mathrm{~cm}$ and $100 \mathrm{~cm}$. The mean angular velocities at 10 degrees retinal eccentricity generated by this driving are 4, 2 and $1 \mathrm{~min}$ of arc per sec respectively. So the velocity for the $100 \mathrm{~cm}$ condition is below, for the $50 \mathrm{~cm}$ condition it is near, and for the $25 \mathrm{~cm}$ condition it is above the thresholds for detection of motion of point lights as measured by Leibowitz and Isabelle (1972).

Postural responses were tested four times for each experimental condition. All conditions in a session were tested in random order for each subject. Each experimental trial lasted for 2 minutes.

\section{Theoretical description of optical flow}

When a subject is fixating straightahead towards a vertical plane from a distance $z$, a pixel in the plane at a distance $x$ from the 
fixation point falls at a retinal eccentricity $\theta$ given by $\theta=\operatorname{arctg}(x / z)$. The retinal velocity (caused both by motion of the pixel itself and by motion of the observer) of that pixel $(\mathrm{d} \theta / \mathrm{d} t)$ induced by a movement of the pixcl relative to the observer in the $z$-direction is given by

$$
\frac{\mathrm{d} \theta}{\mathrm{d} t}=-\cos (\theta) \sin (\theta) \frac{1}{z} \frac{\mathrm{d} z}{\mathrm{~d} t} .
$$

This equation shows that the retinal velocity is proportional to the inverse of the distance to the plane. Similarly, all spatial derivatives of the velocity field (such as rotation, expansion and deformation) decrease with the inverse of the distance (Koenderink and Van Doorn 1976).

This might lead one to the suggestion that postural sway is inversely related to the distance to the scene. However, for the moving scene, if retinal slip is to be minimized, it becomes zero if movements of the observer are equal to the movement of the scene. This predicts that sway amplitude should not depend on distance to the scene.

\section{Results}

Fig. 1 shows the mean power (root mean square) of postural sway in fore-after direction as a function of the distance to the stationary scene for three observers. In an analysis of variance it appeared that postural sway increases with increasing distance to the scene $(F(5,10)$ $=4.37 ; p<0.05)$. These results are in agreement with earlier findings obtained in similar experiments by Brandt et al. (1985) and Paulus et al. (1989).

In the second experiment, sway in response to a sinusoidally moving scene was investigated as a function of distance. Fig. 2 shows the results for five subjects. In $90 \%$ of the trials the power spectrum of postural responses in foreafter direction showed a marked peak at the $0.2 \mathrm{~Hz}$ frequency of modulation. The amplitude of postural sway at the frequency of the simulated motion (for the cases with a clear peak) is shown in fig. 2. As the signal-to-noise ratio at the peak was about 10 it was unnecessary to subtract the noise level. It is obvious that the amplitude of sway in fore-after direction does not decrease with distance. This was confirmed in an analysis of variance which 


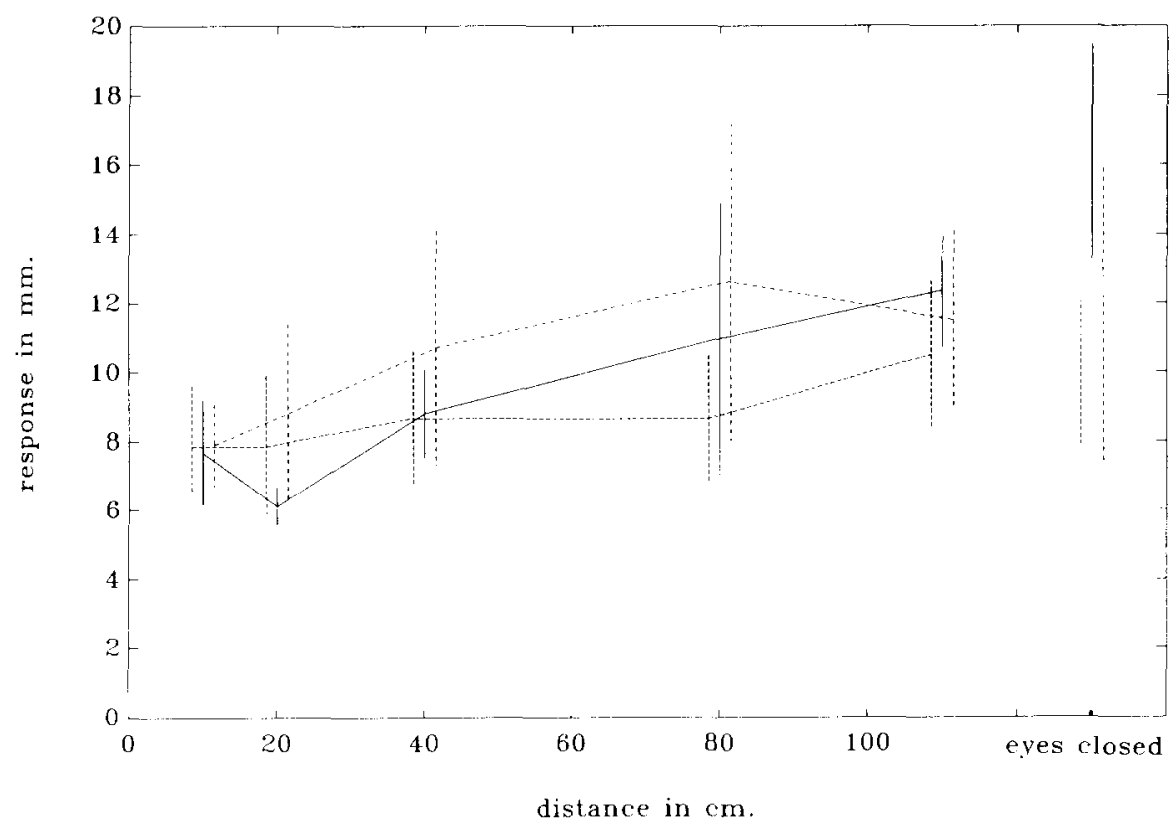

Fig. 1. Mean amplitude of postural sway in fore-after direction as a function of distance between observer and the stationary scene. The different line types refer to data from three different observers and the vertical bars to the standard deviation.

demonstrated neither a significant increase nor decrease of sway amplitude with distance to the scene $(F(2,8)=2.1 ; p>0.1)$.

\section{Discussion}

The main results of this study are that postural sway in fore-after direction in response to a stationary vertical plane with random dots increases with increasing distance to the plane and that the response to the same stimulus moving with small constant amplitude does not decrease with distance in our testing range. These results can be understood if two assumptions are made: (1) there is a threshold for motion detection which may be different from the psychophysical detection threshold; (2) posture is controlled by minimizing the retinal slip of textural elements on the retina. 


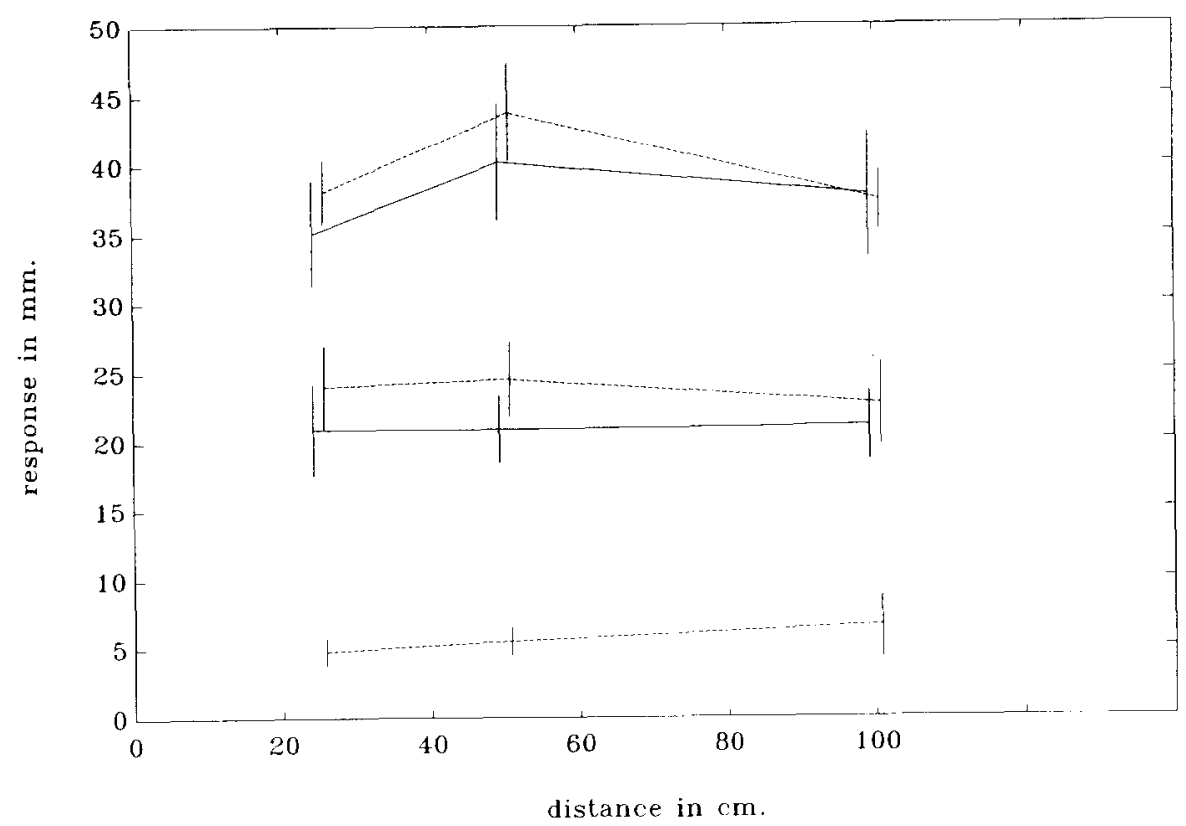

Fig. 2. Sway amplitude in fore-after diraction in response to sinusoidally modulated movement of a vertical plane with an amplitude of $2 \mathrm{~cm}$ along the line of sight of the observers. Different lines refer to data from five different subjects. The amplitude of the frequency component at 0.2 $\mathrm{Hz}$ (the driving frequency) is shown. Standard deviation is shown by vertical bars.

From these assumptions it follows that the sway will be such that the retinal slip is just above threshold. Then it is obvious that the sway increases with distance to the scene. For sway in response to a moving scene the movements of the observer should be approximately equal to the motion of the scene because the retinal slip is already above threshold by the motion of the scene and because retinal slip is minimized by canceling the motion of the scenc.

It has been suggested (Lee 1980) that the time to collision, that is the time needed for an object to reach the subject, plays an important role in the guidance of motor activity. This parameter is proportional to the reciprocal of the amount of expansion in the optic flow field. One can easily show that the time-to-contact increases linearly with distance (Simpson 1988). If one hypothesizes a threshold for time-tocontact and maximization of time-to-contact, one has an equivalent 
explanation for the data. Our data do not discriminate between optic flow theories and time-to-contact theories.

We have no explanation yet for the small slope of the increase of sway for the stationary scene. All theories predict a linear increase in response which is found neither by us nor by Brandt et al. (1985) and Paulus et al. (1989).

Our results do not provide information about the underlying mechanisms that are involved in minimizing retinal slip. One direct way is just to minimize the mean retinal slip, such as proposed by Koenderink and Van Doorn (1987). Another way is to decompose the optic flow in translation, divergence, rotation and deformation components which uniquely define the movement relative to the environment. Postural control then is achieved by minimizing the output of channels that signal the presence of these optic flow components.

\section{References}

Brandt, T., W.M. Paulus and A. Straube, 1985. 'Visual acuity, visual field and visual scene characteristics affect postural balance'. In: B. Igarashi and I. Black (eds.), Vestibular and visual control on posture and locomoter equilibrium. Basel: Karger. pp. 93-98.

Brandt, T., E.R. Wist and J. Dichgans, 1975. Foreground and background in dynamic spatial orientation. Perception and Psychophysics 17, 497-503.

Dichgans, J. and T. Brandt, 1978. 'Visual-vestibular interaction: Effects on self-motion perception and postural control'. In: R. Held, H. Leibowitz and H. Teuber (eds.), Handbook of sensory physiology, Vol. 8. Berlin: Springer-Verlag. pp. 755-804.

Fisher, S.K. and K.J. Ciuffreda, 1988. Accommodation and apparent distance, Perception 17. $609-621$.

Gielen, C.C.A.M. and W.N.J.C. van Asten, 1990. Postural responses to simulated moving environments are not invariant for the direction of gaze. Experimental Brain Research 79 , $167-174$.

Howard, I.P. and T. Heckmann, 1989. Circular vection as a function of the relative sizes, distances, and positions of two competing visual displays. Perception 18, 657-665.

Koenderink, J.J. and A.J. van Doorn, 1987. Facts on optic flow. Biological Cybernetics 56, $247-254$.

Lee, D.N., 1980. 'Visuo-motor coordination in space-time'. In: G.E. Stelmach and J. Requin (eds.), Tutorials in motor behaviour. Amsterdam: North-Holland. pp. 281-295.

Leibowitz, C.J.H. and E. Isabelle, 1972. Peripheral motion detection and refractive error. Science 177, 1207-1208.

Paulus, W., A. Strauhe, S. Krafczyk and T. Brandt, 1989. Differential effects of retinal target displacement, changing size and changing disparity in the control of anterior/posterior and lateral body sway. Experimental Brain Research 78, 243-252.

Simpson, W.A., 1988. Depth discrimination from optic flow. Perception 17, 497-512.

Van Asten, W.N.J.C., C.C.A.M. Gielen and J.J. Denier van der Gon, 1988a. Postural movements induced by rotations of visual scenes. Journal of the Optical Society of America A 5 , 1781-1789. 
Van Asten, W.N.J.C., C.C.A.M. Gielen and J.J. Denier van der Gon, 1988b. Postural responses induced by simulated motion of differently structured environments. Experimental Brain Research 73, 371-383.

Warren, W.H., M.W. Morris and M. Kalish, 1988. Perception of translational heading from optical flow. Journal of Experimental Psychology: Human Perception and Performance 14, $646-660$. 